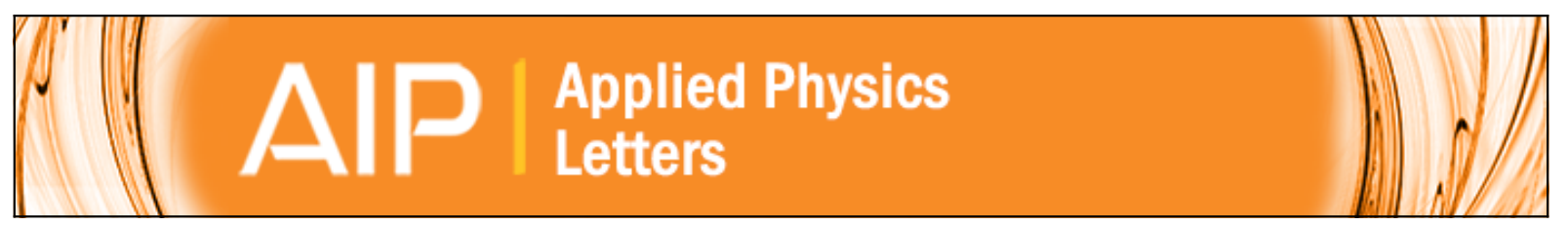

\title{
Improving information density in ferroelectric polymer films by using nanoimprinted
} gratings

D. E. Martínez-Tong, M. Soccio, M. C. García-Gutiérrez, A. Nogales, D. R. Rueda, N. Alayo, F. Pérez-Murano, and T. A. Ezquerra

Citation: Applied Physics Letters 102, 191601 (2013); doi: 10.1063/1.4804427

View online: http://dx.doi.org/10.1063/1.4804427

View Table of Contents: http://scitation.aip.org/content/aip/journal/apl/102/19?ver=pdfcov

Published by the AIP Publishing

\section{Articles you may be interested in}

Nanoscale domain growth dynamics of ferroelectric poly(vinylidene fluoride- co -trifluoroethylene) thin films

Appl. Phys. Lett. 96, 012908 (2010); 10.1063/1.3290247

The effect of defects on the electronic structure of long chain ferroelectric polymers

J. Appl. Phys. 106, 044105 (2009); 10.1063/1.3204490

Low-temperature dielectric properties of Langmuir-Blodgett ferroelectric polymer films

J. Appl. Phys. 103, 034110 (2008); 10.1063/1.2838212

Ferroelectric nanomesa formation from polymer Langmuir-Blodgett films

Appl. Phys. Lett. 85, 3528 (2004); 10.1063/1.1808251

Nonvolatile memory element based on a ferroelectric polymer Langmuir-Blodgett film

Appl. Phys. Lett. 82, 142 (2003); 10.1063/1.1533844

\section{A|P| $\left.\right|_{\text {Applied Physics }} ^{\text {Journal of }}$}

Journal of Applied Physics is pleased to announce André Anders as its new Editor-in-Chief 


\title{
Improving information density in ferroelectric polymer films by using nanoimprinted gratings
}

\author{
D. E. Martínez-Tong, ${ }^{1, a)}$ M. Soccio, ${ }^{1}$ M. C. García-Gutiérrez, ${ }^{1}$ A. Nogales,${ }^{1}$ D. R. Rueda,${ }^{1}$ \\ N. Alayo, ${ }^{2}$ F. Pérez-Murano, ${ }^{2}$ and T. A. Ezquerra ${ }^{1, a)}$ \\ ${ }^{1}$ Instituto de Estructura de la Materia, IEM-CSIC Serrano 119, 121, Madrid 28006, Spain \\ ${ }^{2}$ Instituto de Microelectrónica de Barcelona IMB-CNM (CSIC), Campus UAB, \\ 08193 Cerdanyola del Vallès (Bellaterra), Barcelona, Spain
}

(Received 18 February 2013; accepted 22 April 2013; published online 13 May 2013)

\begin{abstract}
In this work, well-defined low aspect ratio nanostructures based on nanogratings on thin films of poly(vinylidene fluoride-trifluoroethylene) copolymers were prepared. By using these nanogratings, an improved management of writing and reading information of about $500 \mathrm{Gbit} / \mathrm{in}^{2}$ $\left(0.01 \mathrm{bit} / \mathrm{nm}^{2}\right)$ can be reached as revealed by Piezoresponse Force Microscopy. Structural investigation by means of X-ray diffraction techniques indicates that the physical confinement generated by nanoimprint promotes the development of smaller and edge-on oriented crystals. Our results evidence that one-dimensional nanostructuring can be a straightforward approach to improve the control of the polarization in ferroelectric polymer thin films. C 2013 AIP Publishing LLC. [http://dx.doi.org/10.1063/1.4804427]
\end{abstract}

The development of non-volatile organic memories, defined as devices where the information is stored permanently without the requirement of a continuous electrical feed, is currently subject of strong debate. Several approaches and prototypes have already been proposed, for example, metalpolymer-metal capacitors, ${ }^{1-5}$ field-effect transistors, ${ }^{6-9}$ and tunnel-junctions, ${ }^{10}$ all of them based on thin polymer films, generally with thickness smaller than $100 \mathrm{~nm}$, and with the possibility of a readily integration with flexible complementary metal oxide semiconductor devices or other organic electronic devices. ${ }^{11}$ Poly(vinylidene fluoride) (PVDF) and its copolymers with trifluoroethylene, $\mathrm{P}(\mathrm{VDF}-\mathrm{TrFE})$, are mostly proposed as candidates for the development of organic memories since they exhibit excellent ferro, piezo, and pyroelectric properties. Instead of using simple thin films as the main memory component, it has been recently proposed that geometrical structuring of the films could be a significant improvement. This structuring is mostly accomplished by Nanoimprint Lithography (NIL). ${ }^{12-23}$ In principle, the simpler approach would be to use simple P(VDF-TrFE) thin films, which can be easily processed by spin-cast techniques. However, P(VDF-TrFE) thin films typically exhibit roughness levels that make them not suitable for the development of nanocontrolled devices. ${ }^{12}$ As revealed by recent Piezoresponse Force Microscopy (PFM) studies, ${ }^{24}$ the control of the polarization in $\mathrm{P}(\mathrm{VDF}-\mathrm{TrFE})$ thin films can be problematic mainly due to the strong heterogeneity of the crystalline morphology leading to non-uniform local fields which leads to remote domain nucleation and non-uniform spreading of the ferroelectric domains. $^{24}$

Here, we report how the development of nanogratings on thin films of P(VDF-TrFE), prepared by NIL can be a straightforward approach to improve the management of writing and reading high density of information. P(VDFTrFE) copolymer, with a VDF/TrFe composition of 74/26 as

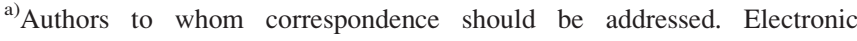
addresses: t.ezquerra@csic.es and d.martinez@iem.cfmac.csic.es
}

revealed by NMR, was obtained from Piezotech S.A.S (France). Polymer thin films were prepared by spin coating on silicon wafers (100) (Wafer World Inc., USA) polished on one surface. The wafers were previously cleaned with acetone and isopropanol, respectively, and dried by nitrogen flow. P(VDF-TrFE) 76/24 was solved in N,N-dimethylacetamide (Sigma-Aldrich) with a concentration of $40 \mathrm{~g} / \mathrm{l}$. A fixed amount of $0.1 \mathrm{ml}$ of polymer solution was deposited by a syringe on a rectangular $\left(2 \times 2 \mathrm{~cm}^{2}\right)$ silicon substrate. An initial acceleration was applied for $4 \mathrm{~s}$ in order to reach a rotational speed of $4800 \mathrm{rpm}$. This final speed was kept for $2 \mathrm{~min}$. Polymer films of thickness about $100 \pm 5 \mathrm{~nm}$, as measured by spectrophotometry (Nanospec 6100) are typically obtained under these conditions. For the sake of comparison, we also investigated the polymer film area outside the nanoimprinted region. We performed NIL at a constant molding temperature and pressure using a thermal nanoimprint machine (Obducat). Silicon stamps were prepared by electron beam lithography, metal deposition lift-off, and reactive ion etching. The nanostructured array on the stamp covers an area of $\left(1.5 \times 1.5 \mathrm{~mm}^{2}\right)$. The resulting height of the lines was $135 \pm 5 \mathrm{~nm}$, as determined by Atomic Force Microscopy (AFM). The arrays of lines and trenches were fabricated on mesa-type silicon stamps to facilitate the imprinting process. The area of the mesa is $2 \times 2 \mathrm{~mm}^{2}$ and $\approx 400 \mathrm{~nm}$ of height and it was defined by means of optical lithography and wet silicon etching. To facilitate stamp separation after embossing, stamps were coated with a monolayer of perfluorodecyltrichlorosilane deposited from the gas phase. During the imprint process, first, the silicon stamp and the polymer coating were heated up to $180^{\circ} \mathrm{C}$ without applying pressure until reaching a steady temperature. Subsequently, the polymer film was nanostructured by applying a pressure of $4053 \mathrm{kPa}$ extruding the softened polymer material to fill the nanocavities in the stamp. Temperature and pressure were kept fixed for $5 \mathrm{~min}$. After this step, the system was cooled down to $50{ }^{\circ} \mathrm{C}$, keeping the applied pressure. After removing the pressure, the system is allowed to gradually cool down to room 
temperature and then the polymer nanostructures were selfdetached from the silicon master stamp.

Polymer gratings were investigated by means of Grazing Incidence X-ray Scattering at Small (GISAXS) and Wide (GIWAXS) Angles at the BW4 beamline (HASYLAB, DESY, Hamburg, Germany). The experimental setup for GISAXS and GIWAXS has been previously described. ${ }^{25-27}$ A scheme of the experimental setup is shown in Fig. 1. Samples are positioned horizontally and aligned with their lines parallel to the X-ray beam. ${ }^{19,28-30}$ The incoming $\mathrm{X}$-ray beam reaches the sample with a certain incidence angle $\alpha_{\mathrm{i}}$. The incident and reflected beams define the vertical scattering plane. ${ }^{31}$ Both the scattering plane and the sample plane intersect the detector along the meridian and the horizon, respectively, which are the reference lines to measure the angles $\omega$ and $\alpha$. The scattering can be interpreted on the basis of the three orthogonal scattering vectors $\mathrm{q}_{\mathrm{x}}=(2 \pi / \lambda)\left(\cos \omega \cos \alpha-\cos \alpha_{\mathrm{i}}\right), \mathrm{q}_{\mathrm{y}}=(2 \pi / \lambda) \sin \omega \cos \alpha$, and $\mathrm{q}_{\mathrm{z}}=(2 \pi / \lambda)\left(\sin \alpha_{\mathrm{i}}+\sin \alpha\right), \lambda$ being the wavelength of the X-ray beam. An X-ray wavelength $\lambda=0.138 \mathrm{~nm}$, with a beam size $(\mathrm{H} \times \mathrm{V})$ of $40 \times 20 \mu \mathrm{m}^{2}$ was used. Scattered intensity was recorded by a Mar CCD detector of $2048 \times 2048$ pixels with a resolution of $79.1 \mu \mathrm{m}$ per pixel, and a sampleto-detector distance of $2.325 \mathrm{~m}$ for GISAXS experiments and of $0.106 \mathrm{~m}$ for GIWAXS. An incidence angle of $\alpha_{\mathrm{i}}=0.4^{\circ}$ was selected. Acquisition times were optimized in order to get maximum number of counts avoiding saturation of the detector. Typical acquisition times of $1 \mathrm{~s}$ for stamps and $5 \mathrm{~s}$ for NIL polymer gratings were used. PFM measurements ${ }^{32}$ were carried out by means of a Nanoscope V AFM (Bruker). The topography and the ferroelectric signals were acquired simultaneously. The microscope was used in contact mode, with a low deflection set-point in order to avoid damaging the samples. The selected probes were the SCM-PIC (Bruker), which have a low spring constant $(0.2 \mathrm{~N} / \mathrm{m})$ and a conductive PtIr layer. Local poling of the films and NIL polymer gratings were carried out using the tip as the top electrode. In every case, a DC bias $\pm 12 \mathrm{~V}$ was applied for $5 \mathrm{~min}$. The PFM out-of-plane signal was taken applying an

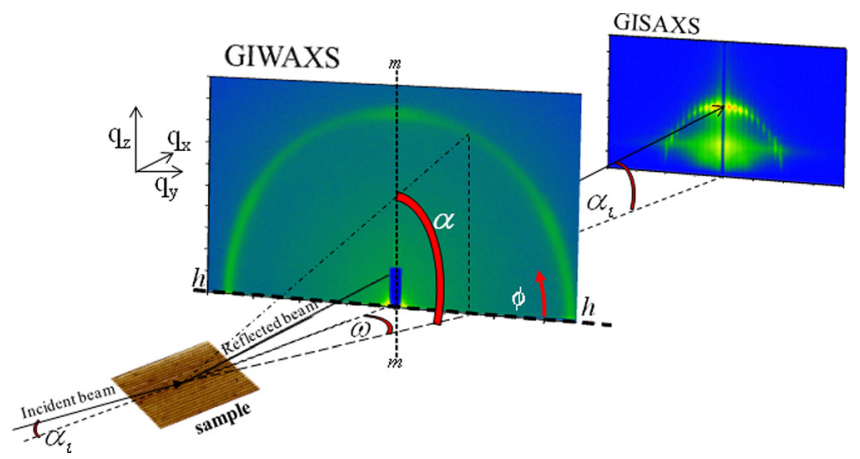

FIG. 1. Scheme of a GIWAXS and GISAXS experiment. The scattering plane, containing both the direct and the specular beams, intersects the 2D detector along the meridian, $m-m$ line. The horizon, $h$ - $h$ line, is the intersection between the sample and the $2 \mathrm{D}$ detector planes that are perpendicular to each other. Each point on the pattern can be characterized by the exit angle, $\alpha$, and the out of scattering plane angle $\omega$. In the detector plane, an azimuth angle $\phi$ can be defined useful to account for oriented patterns. A much longer sample-to-detector distance is required for a GISAXS experiment than for a GIWAXS one. The incidence angle was enlarged for the sake of clarity.
AC voltage of $2 \mathrm{~V}$ at a frequency of $60 \mathrm{kHz}$ between sample and tip. The PFM in-plane response was characterized modulating the photodetector signal through a lock-in amplifier and measuring the changes in the first harmonic. Hysteresis cycles were recorded applying a tip bias ramp from $-12 \mathrm{~V}$ to $12 \mathrm{~V}$ at a frequency of $0.1 \mathrm{~Hz}$. Fig. 2(a) shows an AFM topographic image of a thin film of $\mathrm{P}(\mathrm{VDF}-\mathrm{TrFE})$ of thickness $\approx 100 \pm 2 \mathrm{~nm}$ and roughness values of r.m.s. $\approx \pm 20 \mathrm{~nm}$. Here, one sees the characteristic morphology of P(VDFTrFE) copolymers characterized by random large needlelike crystals, ${ }^{2}$ which has been associated to edge-on lamellae, which coexist with flat-on crystals appearing as irregular flakes on the image. This morphology is corroborated by Scanning Electron Microscopy (SEM) micrographs (Inset of Fig. 2(a)). This morphology is also supported by GIWAXS experiments which delivers the type of patterns included in Fig. 3(a). The GIWAXS pattern for the thin film (Fig. 3(a)) shows the strongest reflections (200) and (110), of similar lattice spacing, observed at $|\mathrm{q}|=13.78 \pm 0.34 \mathrm{~nm}^{-1}$, corresponding to the orthorhombic crystal lattice of $\mathrm{P}(\mathrm{VDF}-\mathrm{TrFE})$ copolymers. ${ }^{2,33,34}$ These two reflections are separated $60^{\circ}$ from one another in a hk0 diffraction pattern. ${ }^{2}$ The intensity distribution on the observed semi-ring (Fig. 3(a)) can be used to characterize both polymer chain and lamellae orientation on these thin films. On one hand, the existence of significant intensity on the equatorial region can be attributed to the presence of flat-on lamellae with polymer chains oriented

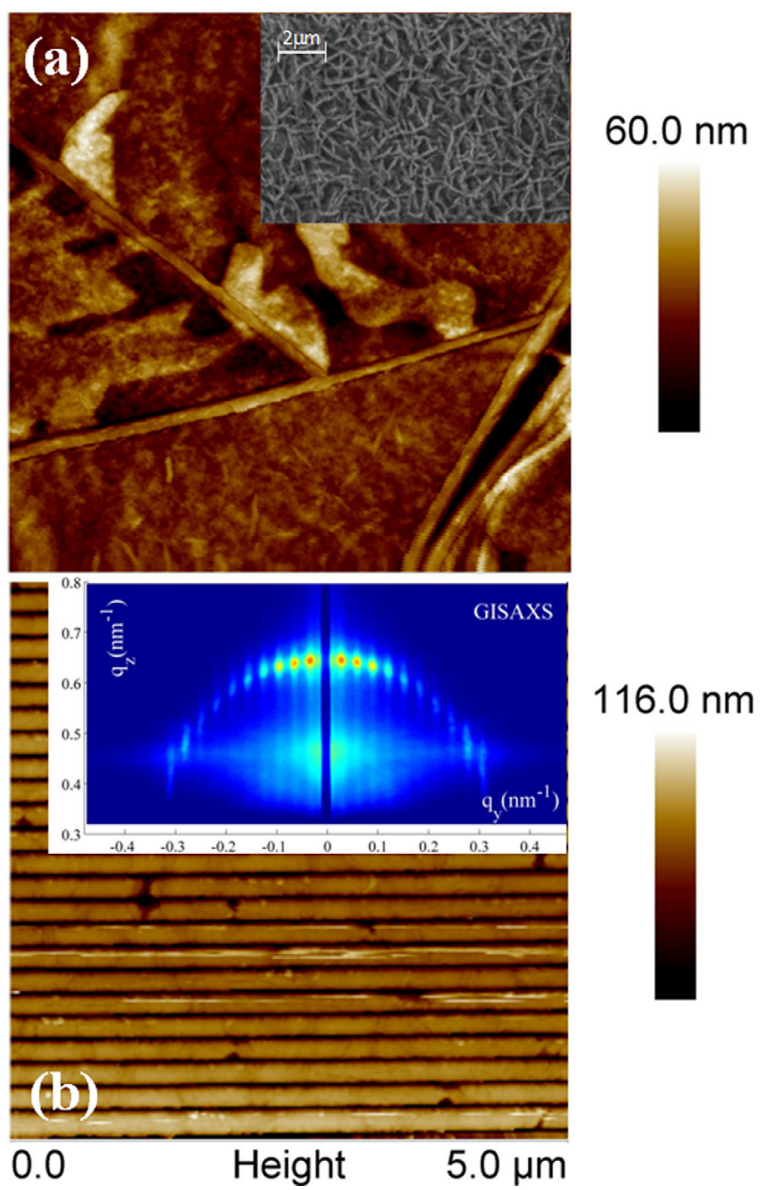

FIG. 2. AFM topographic images of: (a) P(VDF-TrFE) thin film (inset shows a SEM micrograph) and of (b) $\mathrm{P}(\mathrm{VDF}-\mathrm{TrFE})$ grating prepared by NIL. Inset shows a GISAXS pattern. 

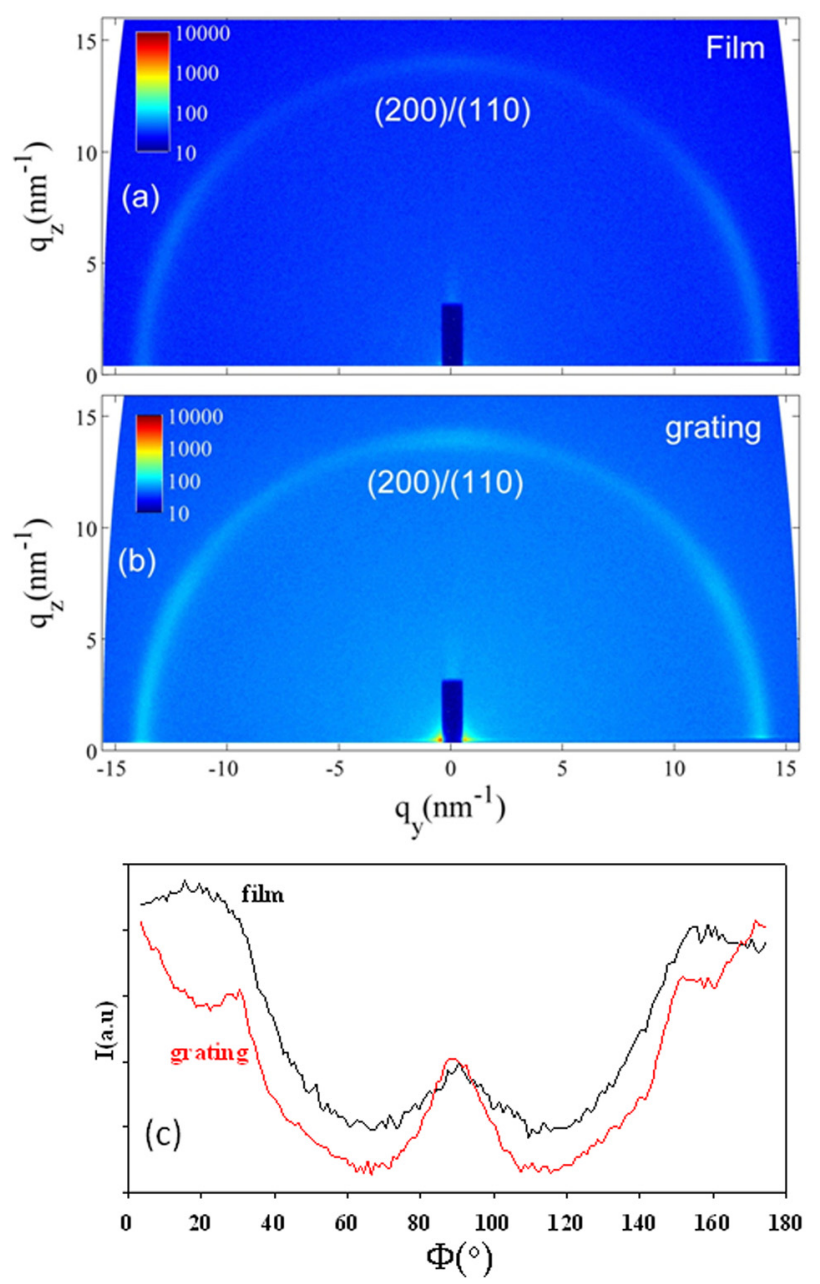

FIG. 3. GIWAXS patterns of: (a) P(VDF-TrFE) thin film and (b) P(VDFTrFE) grating. (c) Azimuth intensity profiles of the multiple reflection (200)/ (110) for both film and grating.

perpendicular to the surface. ${ }^{2}$ On the other hand, a high intensity on the meridian indicates the existence of edge-on lamellae with polymer chains lying on the substrate. ${ }^{9}$ AFM/SEM images and GIWAXS indicate that the P(VDF-TrFE) thin films consist of a mixture of flat-on and edge-on lamellae with some degree of tilting. The topographic height, PFM amplitude, and phase images of a selected region of the film are shown in Figs. 4(a)-4(d), respectively. Fig. 4(c) displays the amplitude profile along the poled line of Fig. 4(b) in comparison with that of the unpoled one. The PFM image was taken after applying a tip poling voltage of $+12 \mathrm{~V}$ for $300 \mathrm{~s}$ over a horizontal line at the centre of the image. Before poling, the PFM amplitude images exhibit essentially no contrast. However, after poling over a horizontal line in the sample that can be visualized at the centre of the image (Fig. 4(b)), the presence of a non-zero amplitude is a clear manifestation of the ferroelectric response of the polymer. It is obvious that after poling the ferroelectric response is not restricted to the poling line but it propagates along extended portions of the needlelike crystals as large as several microns. The strong out-of-plane signal further corroborates that the needlelike crystals mainly consist on edge-on lamellae with polymer chains aligned parallel to the substrate. ${ }^{24}$ Fig. 4(e) displays the hysteresis loops of the PFM phase of both the out-of-plane and the in-plane signals. The hysteresis loops exhibit a clear
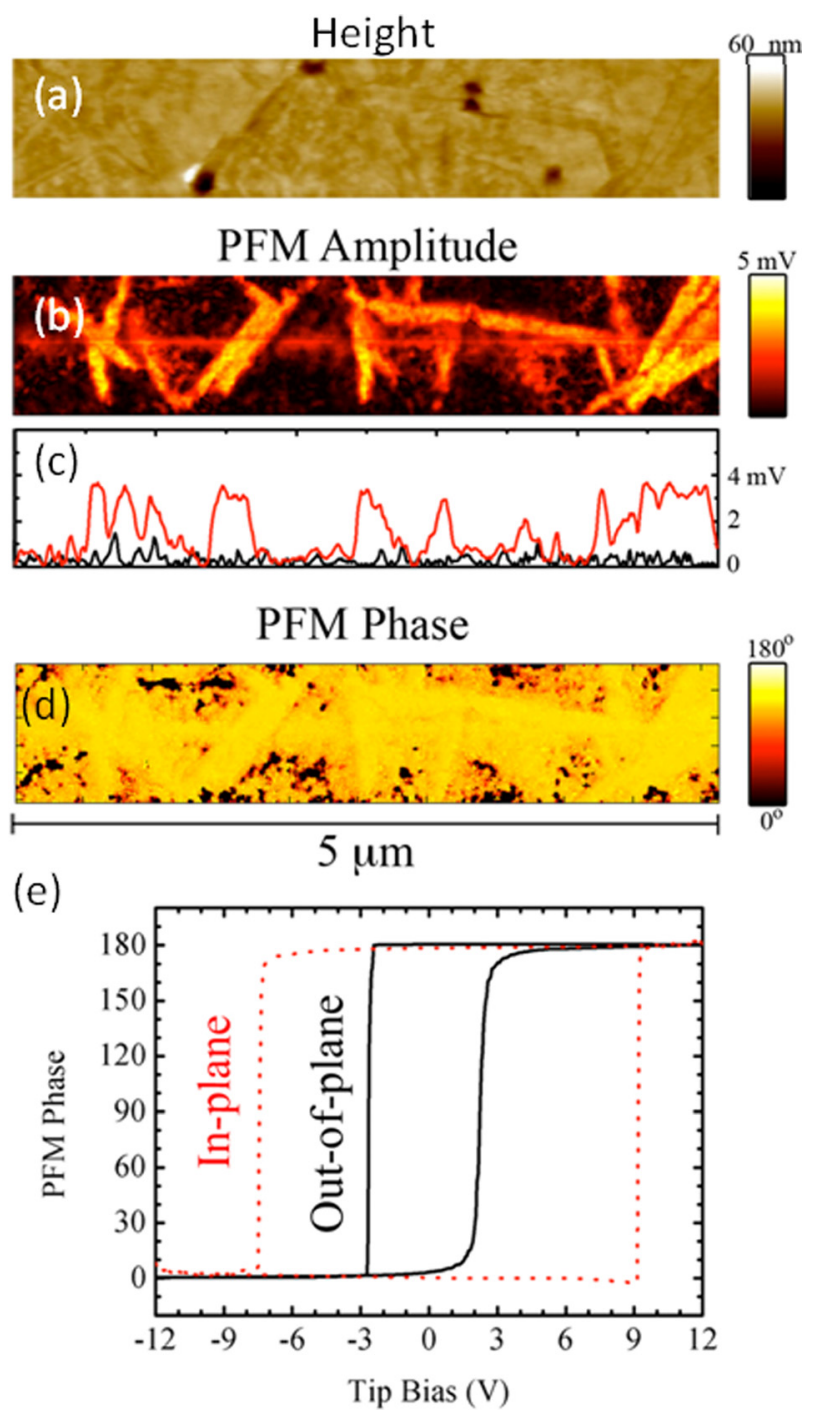

FIG. 4. (a) AFM topographic image of a P(VDF-TrFE) thin film. PFM images for (b) amplitude and (d) phase of the same film area. Fig. 4(c) displays the amplitude profile along the poled line of Fig. 4(b) (red) and that of the unpoled one (black). (e) Hysteresis loops of the PFM phase for both the out-of-plane and of the in-plane contributions.

anisotropy as expected from the previously discussed coexistence of edge-on and flat-on crystals. In both cases, a phase change of $180^{\circ}$ is detected indicating the presence of a flipping process of the permanent dipole moments. Vertical and horizontal ferroelectric domains seem to have a quantitatively different ferroelectric response since a coercive voltage of about $3.0 \pm 0.5 \mathrm{~V}$ is needed to produce the flip in the edge-on lamellae while a higher voltage $\approx 8 \pm 1 \mathrm{~V}$ is required to polarize the flat-on ones. This behavior could be reproduced at several sample locations and reveals the morphological heterogeneity of the system. The differences in coercive fields for vertical and horizontal domains can be qualitatively understood in terms of the molecular motions involved in the flipping process since H-C-F dipole can easily rotate in the edge-on crystals ${ }^{2}$ and can be associated to the out-of-plane PFM signal. On the contrary, the dipole rotation in flat-on crystals is severely hindered due to steric constrains. ${ }^{2}$

Until now, we have shown that it is possible to write information in $\mathrm{P}(\mathrm{VDF}-\mathrm{TrFE})$ spin-coated films $100 \mathrm{~nm}$ thick. However, the uncontrolled large extension of the ferroelectric 


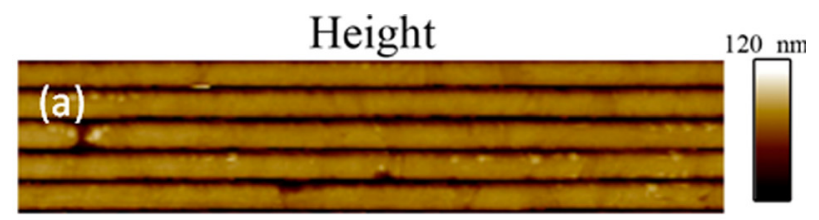

PFM Amplitude
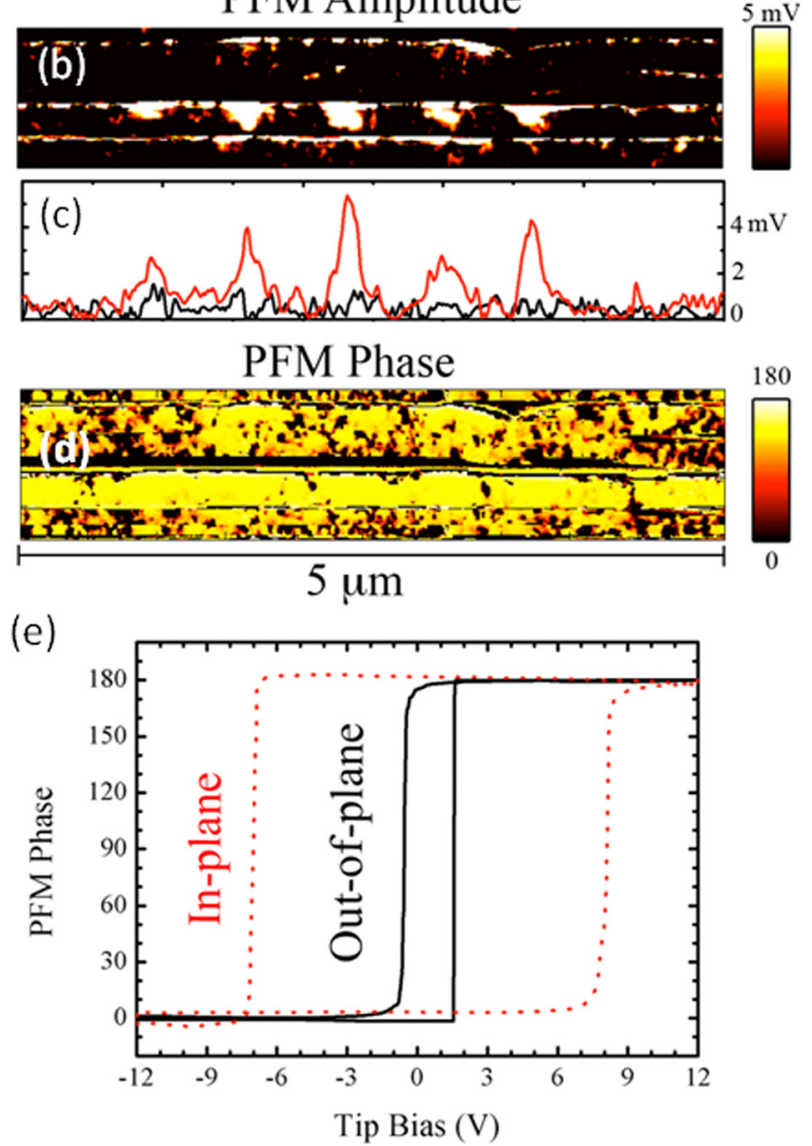

FIG. 5. (a) AFM topographic Image of a P(VDF-TrFE) grating. PFM images for (b) amplitude and (d) phase of the same grating area. Fig. 5(c) displays the amplitude profile of the poled line along a grating line of Fig 5(b) (red) and that of the unpoled grating line (black). (e) Hysteresis loops of the PFM phase of the both the out-of-plane and of the in-plane for the grating.

signal over needlelike crystals can be problematic. Hereafter, we will show how NIL can help to overcome this problem. By means of NIL, it is possible to provide a gratings nanostructure to the P(VDF-TrFE) films (Fig. 2(b)). Here, a grating is shown with a depth of $120 \pm 5 \mathrm{~nm}$, width of $120 \pm 5 \mathrm{~nm}$, and a pitch (line-to-line distance) of $200 \pm 5 \mathrm{~nm}$. The nanoimprinting process allows reducing the r.m.s. roughness to quite low values $\approx \pm 3 \mathrm{~nm}$. A GISAXS pattern of these nanostructured films is shown in Fig. 1 and in the inset of Fig. 2(b) as a function of the q-vectors. The presence in the pattern of a well defined semicircle of sharp spots periodically distributed on it indicates that these gratings can be considered rather close to one-dimensional crystalline lattices. ${ }^{29}$ According to Figs. 3(a) and 3(b), GIWAXS patterns of the gratings are qualitatively similar to those of the films. However, azimuth intensity profiles of the semi-ring reveal subtle differences between films and gratings as shown in Fig. 3(c). In the case of the grating, clear maxima at $30^{\circ}$ and $90^{\circ}$ are resolved. As mentioned above, this feature is characteristic for an edge-on morphology with the polymer chain parallel to the beam. Considering that the $\mathrm{X}$-rays beam impinges the grating along the lines (Fig. 1), this indicates that for the gratings edge-on lamellae are more abundant than in the film. This observation is in agreement with the crystal orientation observed for P(VDF-TrFE) confined within self-assembled organosilicates. ${ }^{9}$ Figs. 5(a)-5(d) show the topographic PFM amplitude and phase images, respectively, of a selected region of the polymer grating. Fig. 5(c) displays the amplitude profile of the poled line along a grating line (Fig. 5(b)) in comparison with that scanned without poling. In this case, the PFM image was taken after applying a tip poling voltage of $+12 \mathrm{~V}$ for $300 \mathrm{~s}$ in 5 spots, over the fourth line from the top visualized in the image. As for the films, before poling, the gratings show no PFM contrast. Since PFM must be carried out in contact mode, the direction of scan was imposed to be parallel to the grating line direction in order to avoid damage of the imprinted lines due to the probesample interactions. After poling, the out-of-plane PFM amplitude exhibits clearly the 5 written points as shown in Fig. 5 (b). The average size of the points is $\approx 250 \mathrm{~nm}^{2}$. It is worth mentioning that the extension of the ferroelectric domains in the gratings is about one order of magnitude smaller than those obtained in the spin-coated films. The ferroelectric nature of the gratings is further characterized by the hysteresis loops of the PFM phase of the both out-of-plane and in-plane signals (Fig. 5(e)). Similarly, as for the films, the hysteresis loops exhibit a clear, expected anisotropy considering the coexistence of edge-on and flat-on crystals on the gratings as well as in the films. The decrease of the ferroelectric domains size in the gratings can be attributed to the limitation of the growth of the needlelike polymer crystals imposed by the wall trenches of the grating. The technological interest of this grating nanostructure for ferroelectric applications lies in the simplicity of fabricating molds based on lines, instead of the squares or dots. Also, this geometry of lines seems to be more versatile since there are no gaps between the written spots, thus, there are not geometrical limitations imposed. On the other hand, since the propagation of the ferroelectric signal depends on the poling time, ${ }^{34}$ it is possible to expect that short times could decrease our spot size almost in a factor of $10{ }^{34,35}$ Considering this possibility, one might expect an information density of about $0.5 \mathrm{Tbit} / \mathrm{in}^{2}{ }^{2}\left(0.01 \mathrm{bit} / \mathrm{nm}^{2}\right)$ by using a line based nanostructured P(VDF-TrFE) film. This information density compares well with that recently reported $^{36,37}$ for organic-based magnetic structures. Thus, our proposal could be of interest towards the miniaturization of all-organic storage devices.

This work was supported by MINECO MAT2009-07789, MAT2011-23455, and MAT-2012-33517. Access to clean room under GICSERV-7 program is grateful acknowledged. The assistance of J. Perlich (HASYLAB) is gratefully acknowledged. D.E.M. and M.S. acknowledge the tenure of a JAE-Pre fellowship and a JAE-Doc contract of CSIC and the FSE.

${ }^{1}$ R. C. G. Naber, P. W. M. Blom, A. W. Marsman, and D. M. de Leeuw, Appl. Phys. Lett. 85, 2032 (2004).

${ }^{2}$ H. J. Jung, J. Chang, Y. J. Park, S. J. Kang, B. Lotz, J. Huh, and C. Park, Macromolecules 42, 4148 (2009).

${ }^{3}$ D. Mao, I. Mejia, H. Stiegler, B. E. Gnade, and M. A. Quevedo-Lopez, J. Appl. Phys. 108, 094102 (2010).

${ }^{4}$ D. Mao, M. A. Quevedo-Lopez, H. Stiegler, B. E. Gnade, and H. N. Alshareef, Org. Electron. 11, 925 (2010). 
${ }^{5}$ M. A. Khan, U. S. Bhansali, and H. N. Alshareef, Adv. Mater. 24, 2165 (2012).

${ }^{6}$ Y. Guo, G. Yu, and Y. Liu, Adv. Mater. 22, 4427 (2010).

${ }^{7}$ T. N. Ng, B. Russo, B. Krusor, R. Kist, and A. C. Arias, Org. Electron. 12, 2012 (2011).

${ }^{8}$ S. K. Hwang, I. Bae, R. H. Kim, and C. Park, Adv. Mater. 24, 5910 (2012).

${ }^{9}$ S. J. Kang, I. Bae, Y. J. Shin, Y. J. Park, J. Huh, S.-M. Park, H.-C. Kim, and C. Park, Nano Lett. 11, 138 (2011).

${ }^{10}$ D. Y. Kusuma and P. S. Lee, Adv. Mater. 24, 4163 (2012).

${ }^{11}$ R. C. G. Naber, K. Asadi, P. W. M. Blom, D. M. de Leeuw, and B. de Boer, Adv. Mater. 22, 933 (2010).

${ }^{12}$ S. J. Kang, Y. J. Park, J. Y. Hwang, H. J. Jeong, J. S. Lee, K. J. Kim, H.-C. Kim, J. Huh, and C. Park, Adv. Mater. 19, 581 (2007).

${ }^{13}$ Z. Hu, M. Tian, B. Nysten, and A. M. Jonas, Nature Mater. 8, 62 (2009).

${ }^{14}$ Y. Liu, D. N. Weiss, and J. Li, ACS Nano 4, 83 (2010).

${ }^{15}$ J.-R. Fang, X. Y. Luo, Z. Ma, Z. K. Shen, Q. Lu, B. R. Lu, G. D. Zhu, X. P. Qu, R. Liu, and Y. F. Chen, Microelectron. Eng. 87, 890 (2010).

${ }^{16} \mathrm{Z}$. Hu and A. M. Jonas, Soft Matter 6, 21 (2010).

${ }^{17}$ J.-R. Fang, Z.-K. Shen, S. Yang, Q. Lu, J. Li, Y.-F. Chen, and R. Liu, Microelectron. Eng. 88, 2033 (2011).

${ }^{18}$ C.-C. Hong, S.-Y. Huang, J. Shieh, and S.-H. Chen, Macromolecules 45, 1580 (2012).

${ }^{19}$ H. Hlaing, X. H. Lu, T. Hofmann, K. G. Yager, C. T. Black, and B. M. Ocko, ACS Nano 5, 7532 (2011).

${ }^{20}$ S. Ducharme and A. Gruverman, Nature Mater. 8, 9 (2009).

${ }^{21}$ P. Vettiger, G. Cross, M. Despont, U. Drechsler, U. Durig, B. Gotsmann, W. Haberle, M. A. Lantz, H. E. Rothuizen, R. Stutz et al., IEEE Trans. Nanotechnol. 1, 39 (2002).

${ }^{22}$ L. Zhang, S. Ducharme, and J. Li, Appl. Phys. Lett. 91, 172906 (2007).

${ }^{23}$ A. K. Tripathi, A. J. J. M. van Breemen, J. Shen, Q. Gao, M. G. Ivan, K. Reimann, E. R. Meinders, and G. H. Gelinck, Adv. Mater. 23, 4146 (2011).
${ }^{24}$ P. Sharma, T. J. Reece, S. Ducharme, and A. Gruverman, Nano Lett. 11, 1970 (2011).

${ }^{25}$ J. Perlich, J. Rubeck, S. Botta, R. Gehrke, S. V. Roth, M. A. Ruderer, S. M. Prams, M. Rawolle, Q. Zhong, V. Korstgens et al., Rev. Sci. Instrum. 81, 105105 (2010).

${ }^{26}$ S. V. Roth, R. Dohrmann, M. Dommach, M. Kuhlmann, I. Kroger, R. Gehrke, H. Walter, C. Schroer, B. Lengeler, and P. Muller-Buschbaum, Rev. Sci. Instrum. 77, 085106 (2006).

${ }^{27}$ A. Timmann, R. Dohrmann, T. Schubert, H. Schulte-Schrepping, U. Hahn, M. Kuhlmann, R. Gehrke, S. V. Roth, A. Schropp, C. Schroer et al., Rev. Sci. Instrum. 80, 046103 (2009).

${ }^{28}$ J. J. Hernandez, D. R. Rueda, M. C. Garcia-Gutierrez, A. Nogales, T. A. Ezquerra, M. Soccio, N. Lotti, and A. Munari, Langmuir 26, 10731 (2010).

${ }^{29}$ D. R. Rueda, I. Martin-Fabiani, M. Soccio, N. Alayo, F. Perez-Murano, E. Rebollar, M. C. Garcia-Gutierrez, M. Castillejo, and T. A. Ezquerra, J. Appl. Crystallogr. 45, 1038 (2012).

${ }^{30}$ S. Stille, C. Baeumer, S. Krannich, C. Lenser, R. Dittmann, J. Perlich, S. V. Roth, R. Waser, and U. Klemradt, J. Appl. Phys. 113, 064509 (2013).

${ }^{31} \mathrm{P}$. Müller-Buschbaum, in Lecture Notes in Physics, edited by T. A. Ezquerra, M. C. García-Gutiérrez, A. Nogales, and M. Gómez (SpringerVerlag, Berlin, 2009), Vol. 776.

${ }^{32}$ D. A. Bonnell, S. V. Kalinin, A. L. Kholkin, and A. Gruverman, MRS Bull. 34, 648 (2009).

${ }^{33}$ Y. J. Park, S. J. Kang, C. Park, B. Lotz, A. Thierry, K. J. Kim, and J. Huh, Macromolecules 41, 109 (2008).

${ }^{34}$ J. S. Lee, A. A. Prabu, and K. J. Kim, Polymer 51, 6319 (2010).

${ }^{35}$ C. Rankin, C.-H. Chou, D. Conklin, and D. A. Bonnell, ACS Nano 1, 234 (2007).

${ }^{36}$ S. Park, D. H. Lee, J. Xu, B. Kim, S. W. Hong, U. Jeong, T. Xu, and T. P. Russell, Science 323, 1030 (2009).

${ }^{37}$ Z. M. Al-Badri, R. R. Maddikeri, Y. Zha, H. D. Thaker, P. Dobriyal, R. Shunmugam, T. P. Russell, and G. N. Tew, Nat. Commun. 2, 482 (2011). 